
\title{
25 Research Square \\ Estimation of arm kinematics in stroke survivors using wearable sensors
}

\section{Jeffrey Z. Nie ( $\square$ jnie31@siumed.edu )}

Southern Illinois University School of Medicine https://orcid.org/0000-0001-5213-4252

\section{James W. Nie}

University of Illinois College of Medicine at Chicago: University of Illinois at Chicago College of Medicine

\section{Na-Teng Hung}

Northwestern University Feinberg School of Medicine

\section{R. James Cotton}

Shirley Ryan AbilityLab

\section{Marc W. Slutzky}

Northwestern University Feinberg School of Medicine

\section{Research Article}

Keywords: Stroke, stroke rehabilitation, wearable sensors, kinematics, inertial measurement units, virtual reality, upper extremity kinematics

Posted Date: March 3rd, 2021

DOl: https://doi.org/10.21203/rs.3.rs-291041/v1

License: (c) (i) This work is licensed under a Creative Commons Attribution 4.0 International License. Read Full License 


\section{Abstract}

\section{Background}

Stroke is the leading cause of long-term disability in the United States, often resulting in upper extremity (UE) motor impairment. Most existing outcome metrics of UE function in rehabilitation are insensitive to change or subject to observer bias. There is growing interest in using movement kinematics to measure UE motor function, since they can provide high-resolution, quantitative measurements. However, measuring arm kinematics in stroke survivors, particularly in the hospital or clinic, can be challenging for traditional optical tracking systems due to non-ideal environments, expense, and a limited ability to perform required calibration poses. The aim of this study was to develop a general framework for accurate measurements of wrist position during reaching movements in people with stroke using relatively inexpensive wearable sensors.

\section{Methods}

We developed and presented two methods, one using inertial measurement units (IMUs) and using virtual reality (Vive) sensors, that practically estimate wrist position with respect to the shoulder. We then assessed the estimation accuracies of each method during a 3D reaching task by using a Vicon motion capture system. We also demonstrated each methods ability to track two kinematic metrics, sweep area and smoothness, in chronic stroke survivors. We computed Pearson correlation coefficients when appropriate.

\section{Results}

Compared to a traditional optical system, both systems tracked with high accuracy during 3D reaching, with mean absolute errors of $1.00 \pm 0.80 \mathrm{~cm}$ and $1.09 \pm 0.51 \mathrm{~cm}$ for the IMU and Vive, respectively. Furthermore, both methods' estimated kinematics highly agreed with each other $(p<0.01)$.

\section{Conclusions}

These methods may be useful for developing kinematic metrics to evaluate stroke rehabilitation outcomes in both laboratory and clinical environments.

\section{Trial Registration}

The clinical trial (ClinicalTrial.gov ID: NCT03401762) was registered on January 17, 2018 (https://clinicaltrials.gov/ct2/show/NCT03401762) and posted on January 1, 2018. The trial is scheduled to be completed by August 2023. The trial was updated on December 2, 2020 and is currently recruiting.

\section{Introduction}


Stroke is a leading cause of disability in the United States. Each year, approximately 795,000 people experience a stroke, adding to the approximately 7.0 million adult stroke survivors[1]. The most common deficit among stroke survivors is impairment of arm or hand movement, with more than $80 \%$ of stroke survivors experiencing this condition acutely and more than $40 \%$ chronically, despite standard rehabilitation[2]. Many outcome measures have been developed in an attempt to evaluate the efficacy of stroke rehabilitation methods[3]. For evaluation of motor function or impairment, several of these outcome measures, such as the Action Research Arm Test (ARAT) and Fugl-Meyer Assessment of Upper Extremity (FMA-UE), involve an observer scoring the patient's ability to perform certain tasks[4,5]. Despite widespread clinical use, most of these motor outcome measures are subject to observer bias and/or are insensitive to small changes in movement quality[6], particularly for more severely impaired patients. Also, most functional outcome measures do not account for compensatory movements, such as movement of the trunk to reach farther, and therefore do not distinguish between compensatory movements or restitution of neural function as the explanation for functional changes[6,7]. These limitations have resulted in a growing interest in the use of kinematic assessments as outcome measures, as they can provide objective, high-resolution quantitative measurements[8-10]. Indeed, a recent position paper called for more routine use of kinematic measures in stroke trials while highlighting the lack of validated and easy-to-use techniques as a key obstacle to this routine use[11].

A number of kinematic assessments have been studied to assess upper limb motor performance in stroke survivors[6,8-10,12-16]. Depending on the task, certain kinematic measures may be more informative than others. For many tasks, tracking pose (3D position and orientation) over time provides a way to directly evaluate motor function. Furthermore, pose over time provides information from which many more kinematic metrics can be derived, such as movement speed, accuracy, smoothness, and range of motion. Determining a person's pose involves estimating relevant joint angles and limb positions. The gold standard for pose estimation is a marker-based optical tracking system, such as the Vicon and OptiTrack motion capture systems[17]. However, these systems are expensive and require careful setup and calibration[18,19]. For the Vicon, complex static poses (e.g., T-pose, with both arms abducted and elbows straight) and dynamic range-of-motion calibration may be required depending on the skeleton model and desired task[20,21]. These movements would be difficult for many stroke survivors to achieve, given frequent inability to extend the impaired elbow. Additionally, cameras have a long setup time[21], and a large laboratory space free of vision-occluding barriers is required to accommodate the many carefully-positioned cameras. These prerequisites make the routine use of these systems to track spatial pose in stroke survivors difficult, suboptimal, and infeasible for most hospital settings. Although markerless tracking systems offer the potential for less expensive setups[22,23], these can have difficulty in measuring 3D movement, often require multiple, relatively expensive high-speed cameras, and have yet to be rigorously evaluated for accuracy. Moreover, as more rehabilitation research studies are beginning in the inpatient setting, there is a need for more portable solutions.

Inertial measurement units (IMUs) are small and easy to set up, and many approaches of varying complexity have used them to track motion in humans[24-32]. Although a full review is beyond the scope of this article, the goal of many of these approaches is to estimate joint angles and/or limb 
positions by utilizing the raw IMU measurements from multiple locations on the body. These raw measurements often include magnetometer, accelerometer, and gyroscope readings. However, IMUs have some disadvantages, such as susceptibility to magnetic field distortion, that can cause substantial error[33].

Virtual reality (VR) systems provide another possible method for estimating human pose, as they inherently are designed to do this when constructing a virtual world. Here, we take advantage of this design in a consumer VR system, the HTC Vive (HTC and Valve). This system consists of small, lightweight, infrared-emitting cubes, called lighthouses, that define a trackable area (i.e., play area). The pose estimates of any Vive headset, controller, or tracker, each of which contains an array of strategically placed photodiodes, in this area are computed and tracked by the VR system. When using the trackers, the Vive was capable of estimating positions and orientations that agree with estimations made by a more expensive, marker-based optical tracking system (Vicon)[34]. Because the Vive is designed as a portable consumer device, this VR system can be used to track human pose in a variety of environments without being susceptible to magnetic fields as are IMUs, at the expense of a slightly longer setup time requirement.

Here, we present and compare two methods, one using IMUs and one using the Vive, for tracking the 3D wrist position with respect to the shoulder in stroke survivors. While some commercial IMU systems provide end-point position in Cartesian space (Xsens), they are expensive. Here, our IMU-based method can be used with any generic IMU that provides magnetometer, accelerometer, and gyroscope measurements. Additionally, by tracking position relative to the shoulder, compensatory trunk movements are factored out, and a true assessment of arm mobility can be obtained. Further, we have made our code that estimates wrist position relative to the shoulder using each method publicly available (see Code Availability). We also derive example kinematic metrics from the wrist position estimations to demonstrate the utility of these mobile methods.

\section{Methods}

All procedures were approved by the Northwestern University Institutional Review Board. To evaluate the accuracy of each tracking method, we recorded data using the IMUs, Vive, and Vicon from one healthy participant. To demonstrate the utility of the IMUs and Vive, we also recorded data from eight participants with stroke using these sensors in the laboratory during reaching tasks (detailed in System Evaluation).

\section{Common Background}

We provide a general overview of the common and specific aspects of both the IMU- and Vive-based methods in the following sections, starting with the commonalities. Further details are in the Appendix. Prior to taking any sensor measurements, we performed the following critical steps to help ensure proper estimation of wrist position: measuring the length of the arm and forearm and aligning the sensors on the limbs. We measured the arm from the acromion to the antecubital fossa, and we measured the forearm from the antecubital fossa to the center of the ventral aspect of the wrist. We placed the arm and 
forearm sensors proximal to the elbow and wrist, respectively, and aligned one axis of each sensor with the long axis of the respective limb (Figure 1a). This step ensured that the directions of the arm and forearm were always known, regardless of each limb's orientation in space.

After taking sensor measurements, we determined the 3D orientations of the arm and forearm sensors to estimate each limb's 3D orientation. We then used these quantities, as well as the measured limb lengths, to construct a serial kinematic chain model that models the upper extremity (UE) and estimates the wrist position with respect to the shoulder, denoted as the position vector Pwriw. The general characteristics and recording procedures for each method are discussed below.

\section{IMU-based Tracking}

For the IMU-based method, we used a 9-axis IMU (Figure 1b) containing a 3-axis accelerometer, 3-axis magnetometer, and 3-axis gyroscope (Trigno IM Sensor, Delsys Inc.) to record raw inertial data. We imported the raw IMU data into MATLAB and resampled the accelerometer and gyroscope data to the magnetometer's sampling frequency of $74 \mathrm{~Hz}$ for analysis.

The accelerometer measures the summed acceleration component from gravity and inertial acceleration in the world frame. The gravity component, facing directly down, and the magnetic measurements, which point in the direction of the Earth's magnetic field, can provide a stable reference frame to define a zero orientation for orientation estimation. However, these are susceptible to some distortions that must be accounted for. For accelerometers, non-gravitational accelerations (e.g., ballistic movements) partially mask gravity. In motor-impaired stroke survivors, this distortion is usually small due to the relative strength of gravitational acceleration and general inability to produce large non-gravitational accelerations. Many metal alloys in nearby objects, such as in walls, floors, beds, and tables, can distort the magnetic field and thus orientation estimates[33]. Because these distortions can have magnitudes of a substantial fraction of the Earth's magnetic field, the magnetometer is much more susceptible to measurement distortion than the accelerometer. Therefore, it is critical to calibrate the magnetometer whenever the local magnetic environment changes. We calibrated the magnetometer by recording while rotating the IMUs by $90^{\circ}$ increments four times about the sensor's positive and negative $x, y$, and $z$ axes, which typically took approximately three minutes. We performed this calibration before putting the IMUs on the arm and forearm. We then used a least-squares ellipsoid fitting method to find the parameters that transformed the recorded magnetometer measurements into a sphere[35,36]. Afterwards, we used the recorded accelerometer recordings to calibrate the accelerometer and remove any cross-axis misalignment between the accelerometer and magnetometer sensor axes[37].

Following calibration, we fused the accelerometer, gyroscope, and magnetometer measurements to estimate sensor orientation by implementing a modified version of the improved explicit complementary filter[24,28]. Complementary filters (CFs) are widely used to compute orientation using 9-axis IMU measurements[24,28-30] because they select only the low frequency, stable components of the accelerometer/magnetometer estimations and the high frequency, drift-free components of the gyroscope 
estimations. We used this specific version due to several important features, including no singularities, flexible gain selection, gyroscope bias compensation, and decoupled magnetometer influence from roll and pitch estimations[24,28]. In general, higher filter gain increases the cutoff frequency, which weighs the accelerometer/magnetometer estimations more, reducing signal drift but introducing more noise, whereas lower gain weighs the gyroscope estimations more, reducing noise but increasing drift. We generally selected the lowest possible gains after considering the calibration and raw measurement quality.

A typical recording session using the IMU proceeded as follows. Prior to recording participant data, we calibrated the sensors in the testing environment. Afterwards, we performed the limb measurement and sensor alignment steps as described in Common Background. At the start of data collection, the participant performed a brief calibration procedure necessary for developing the kinematic chain model (i.e., data calibration procedure, see Appendix for details). This consisted of a static, neutral pose for 3060 seconds orientation estimations for baseline orientation removal, followed by passive flexionextension (FE) four times without pronation-supination (PS) motion. After the data calibration procedure, the participant relaxed and then performed the assessment tasks.

\section{Vive-based Tracking}

In this study, we used first generation lighthouses and trackers. Each Vive lighthouse sweeps alternating horizontal and vertical IR lasers that are detected by the photodiode-containing trackers (Figure 1c) within the play area. The time delay from the onset of each sweep emitted from the fixed lighthouses to detection by the photodiodes allows determination of the position and orientation of any tracker in the play area. For evaluating arm kinematics, the required play area is relatively small $(1.5 \times 1.5 \mathrm{~m})$ since participants can perform arm movements while standing or sitting in a fixed location.

To estimate elbow angle using the Vive, we positioned the lighthouses such that the trackers were always visible to them. A common acceptable setup was having two lighthouses approximately two meters apart face each other on an oblique plane relative to the patient. This was critical to obtain valid pose estimations from the Vive, as failure to do so would increase the chance of occlusion (i.e., lighthouse could not detect the trackers) and therefore introduce potentially large, unpredictable error in pose estimation. Since the required play area was relatively small, it was easy to find an appropriate lighthouse setup. Once placed on the limb, each tracker had an associated position and orientation estimation, expressed with respect to a common world frame. We neglected the position estimations because they can sometimes become unstable during movement[38]. Using the SteamVR Unity Plugin (found in the Unity assets store), we loaded virtual representations of the Vive trackers into Unity 3D to observe their position and orientation in real time. We then extracted and recorded the orientation estimations of each tracker, as well as time stamps synchronized with the computer's clock because the sampling rate varied with the frame rate of Unity, into text files using the Unity Scripting Application Programming Interface. We subsequently imported these files into MATLAB and interpolated the data to a sampling rate of $50 \mathrm{~Hz}$. 
A typical recording session using the Vive proceeded as follows. We first positioned two lighthouses to capture a sufficiently large play area. We confirmed this by moving the trackers in the play area and observing the real-time movement of their virtual representations in Unity without recording data. If the virtual representations ceased to move simultaneously with tracker movement, signifying tracker occlusion, we adjusted the position of the lighthouses until they redetected the trackers. Afterwards, we performed the limb measurement and sensor alignment steps as described in Common Background. Because there was no required data calibration step, the participant simply proceeded with the assessment once the data recording began.

\section{System Evaluation}

For each task, we report the endpoint distance (EPD)-i.e., the scalar distance from the shoulder to the wrist computed as the magnitude of the position vector Pwri ${ }^{\mathbf{w}}$-estimated by the IMU-based and Vivebased methods.

The first task (reaching task) assessed each method's estimation accuracy in comparison to the gold standard Vicon motion capture system. The Vicon system was set up in an enclosed room with eight Vicon Vantage cameras surrounding the workspace. We calibrated the cameras using the system's specified procedure[21]. We placed reflective markers corresponding to the Vicon UE model onto a healthy human participant (Figures 2a and 2b)[20]. This participant then performed a calibration procedure (static pose with shoulder abducted and elbow extended, Figure 2a) optimized for the Vicon system prior to performing the desired task[20,21]. All Vicon data were sampled at $200 \mathrm{~Hz}$ and imported into MATLAB for analysis.

Due to signal interference between the Vicon Vantage cameras and our first generation Vive lighthouse and tracker setup, it was impossible for us to record valid EPD estimations from the Vicon and Vive simultaneously. Therefore, we designed a sequence of multiplanar reaching movements that a healthy human participant could reliably replicate across recording sessions. Specifically, the participant stood at a fixed position relative to a platform with six different marked targets within reaching distance (Figure 3). Starting with the UE extended and at the side, the participant reached towards and touched the first target with the tip of the third finger, then maintained the reach without any radial or ulnar deviation for several seconds, and subsequently returned the UE to the initial position. This sequence was repeated for the remaining five targets. We averaged all samples while maintaining a reach at a target to estimate the EPD at that target.

We collected data from this healthy participant across four different recording sessions: one with Vicon (four reaches to target 1, five reaches to remaining targets, one with both IMU and Vicon (five reaches to all targets), one with both IMU and Vive (four reaches to all targets), and one with Vive (five reaches to all targets). The combined Vicon data contained nine reaches to target 1 and 10 reaches to each remaining target (59 total reaches). We averaged the estimated EPDs per target to obtain the ground truth EPD estimation for each target. We also computed the active range of motion (AROM), defined as the 
difference between the EPD prior to a reach and the EPD at the target, for all reaches per target and averaged them to obtain the ground truth AROM for each reach. Next, we determined the IMU and Vive's estimated EPD at each target for all 54 (nine per target) reaches and subtracted them from the corresponding ground truth EPD. Likewise, we computed the IMU and Vive's estimated AROM for each reach and subtracted them from the corresponding ground truth AROM. We then computed the mean and standard deviation of the absolute values of these differences in estimated EPD and AROM. Finally, we computed the differences in the estimated mean AROM between the reaches to targets one, two, and three for all three methods. These differences allowed us to measure and compare how sensitive each method was at detecting changes in AROM in a given direction and simulated how the methods could detect improved AROM from a course of therapy.

The second task (sweep task) demonstrated each method's ability to provide data to derive clinically relevant kinematic metrics in stroke survivors. We placed IMUs and Vive trackers on the limbs of eight chronic stroke survivors with UE motor impairment (Figure 2c) enrolled in an ongoing clinical trial (NCT03401762) investigating a six-week training protocol of myoelectric computer interface therapy for stroke[12,39]. Four participants performed this task once, and four participants performed this task at two different times (one on weeks 4 and 6 of training, one on weeks 0 and 4, and two on weeks 0 and 6 ). Starting with the affected hand resting on the ipsilateral thigh while sitting, the participant attempted to abduct the affected shoulder to $90^{\circ}$ while extending the affected elbow to $180^{\circ}$, then subsequently horizontally swept to the contralateral side (internally rotated the shoulder) as far as possible. This was repeated three times, yielding three "sweeps" per session. If performed by a motor-intact person, each sweep should trace out a semicircle in the horizontal plane with a radius equal to the sum of the arm and forearm lengths, as the elbow should be fully extended throughout each sweep.

We derived two different clinically relevant metrics from the wrist position: horizontal sweep area and smoothness. We computed the horizontal sweep area by projecting Pwriw onto the horizontal plane and then using MATLAB's polyarea function. This horizontal sweep area is a task-specific scalar and serves as an example kinematic metric that can be derived from the wrist position. Furthermore, the sweep area is effectively a 2D range of motion to all sides of the body, or effectively a "workspace," which makes it a functionally relevant measure[40]. We also estimated the smoothness of each sweep, a measure characteristic of unimpaired movements that has been shown to increase with stroke recovery[41,42]. After differentiating all Pwriw estimations from the initiation (relaxed state immediately prior to shoulder abduction) to termination (relaxed state immediately after maximal shoulder internal rotation) of the sweep using smooth noise-robust differentiators[43], we computed the magnitude of resulting 3D velocity vector to obtain the endpoint speed (EPS). Afterwards, we extracted the peak envelope of the EPS using MATLAB's envelope function (spline interpolation over local maxima) to reduce noise introduced by filter gain selection and numerical differentiation while maintaining the overall shape of the speed profile. We applied the same peak envelope to both the IMU and Vive data. From the peak envelope of the EPS, we computed the normalized mean endpoint speed (i.e., smoothness), defined as the max EPS divided by the mean EPS during the sweep[41]. After estimating both kinematic metrics for each sweep, we calculated 
and compared the mean of each metric over all sweeps. We computed Pearson's correlation coefficient between the IMU and Vive's estimated mean for each metric. For the four chronic stroke participants that performed the task at two different times, we also computed the change over time in the estimated metrics for each method and compared them.

\section{Results}

We investigated the accuracy of the IMUs and Vive by comparing EPD estimations when reaching out to six different targets to those of a Vicon optical tracking system. We also compared each method's estimated sweep area and smoothness during the sweep task.

Both IMUs and Vive tracked the EPD with performance similar to the Vicon throughout the reaching task (Figure 4). Both methods' estimates of EPD and AROM remained within several $\mathrm{cm}$ of the Vicon estimates (Table 1). Across all reaches, the mean absolute errors in EPD for the IMU and Vive were $1.00 \pm$ $0.80 \mathrm{~cm}$ and $1.07 \pm 0.49 \mathrm{~cm}$, respectively. The maximum absolute errors in EPD across all reaches for the IMUs and Vive were $2.74 \mathrm{~cm}$ and $2.88 \mathrm{~cm}$, respectively. Furthermore, the mean absolute error in AROM across all reaches for the IMU and Vive were $2.20 \pm 1.23 \mathrm{~cm}$ and $2.23 \pm 0.87 \mathrm{~cm}$, respectively. The maximum absolute errors in AROM across all reaches for the IMUs and Vive were $4.80 \mathrm{~cm}$ and $4.29 \mathrm{~cm}$, respectively. Moreover, both the IMU and the Vive were very accurate in estimating changes in AROM (DAROM, Table 2). The absolute error in DAROM for IMU and Vive (compared to Vicon) were $0.73 \pm 0.96$ $\mathrm{cm}$ and $0.36 \pm 0.52 \mathrm{~cm}$, respectively. This is important, as this is the type of measurement that is most relevant to rehabilitation outcomes (e.g., improvement in reaching due to a therapy).

Table 1. Estimation accuracy of the IMU and Vive during the first task.

\begin{tabular}{|c|c|c|c|c|c|c|}
\hline \multirow[t]{2}{*}{ Target } & \multirow[t]{2}{*}{ Vicon EPD (cm) } & \multirow[t]{2}{*}{ Vicon AROM (cm) } & \multicolumn{2}{|c|}{ Absolute EPD error $(\mathrm{cm})$} & \multicolumn{2}{|c|}{ Absolute AROM error (cm) } \\
\hline & & & IMU & Vive & IMU & Vive \\
\hline Target 1 & $38.61 \pm 0.28$ & $14.77 \pm 0.26$ & $1.62 \pm 0.97$ & $1.24 \pm 0.48$ & $3.23 \pm 1.57$ & $2.76 \pm 0.44$ \\
\hline Target 2 & $45.74 \pm 0.54$ & $7.54 \pm 0.61$ & $0.67 \pm 0.47$ & $1.24 \pm 0.39$ & $2.30 \pm 0.82$ & $2.67 \pm 0.41$ \\
\hline Target 3 & $50.80 \pm 0.23$ & $2.47 \pm 0.25$ & $0.35 \pm 0.29$ & $0.79 \pm 0.31$ & $2.14 \pm 0.33$ & $2.23 \pm 0.21$ \\
\hline Target 4 & $42.13 \pm 0.47$ & $11.21 \pm 0.47$ & $1.20 \pm 0.74$ & $1.47 \pm 0.65$ & $3.19 \pm 0.84$ & $2.95 \pm 0.62$ \\
\hline Target 5 & $51.18 \pm 0.18$ & $2.13 \pm 0.28$ & $0.30 \pm 0.17$ & $0.75 \pm 0.05$ & $2.03 \pm 0.27$ & $2.17 \pm 0.05$ \\
\hline Target 6 & $50.13 \pm 0.52$ & $3.31 \pm 0.58$ & $1.84 \pm 0.23$ & $0.95 \pm 0.50$ & $0.29 \pm 0.25$ & $0.61 \pm 0.40$ \\
\hline
\end{tabular}

Vicon endpoint distance (EPD) and active range of motion (AROM) are the means of the Vicon's estimations across all nine reaches per target. Absolute EPD/AROM errors are the mean of the absolute differences between the Vicon EPD/AROM and the IMU and Vive EPD/AROM estimations for all reaches per target.

Table 2. Estimation sensitivity of the IMU and Vive during the first task. 


\begin{tabular}{cccc}
\hline & Vicon $\triangle$ AROM $(\mathrm{cm})$ & IMU $\triangle$ AROM $(\mathrm{cm})$ & Vive $\Delta$ AROM $(\mathrm{cm})$ \\
\hline Target $1 \rightarrow$ Target 2 & $-7.23 \pm 0.66$ & $-6.30 \pm 1.77$ & $-7.14 \pm 0.60$ \\
Target $1 \rightarrow$ Target 3 & $-12.30 \pm 0.36$ & $-11.21 \pm 1.60$ & $-11.77 \pm 0.48$ \\
Target $2 \rightarrow$ Target 3 & $-5.07 \pm 0.65$ & $-4.91 \pm 0.88$ & $-4.63 \pm 0.46$ \\
\hline
\end{tabular}

$\triangle \mathrm{AROM}$ is the difference between the mean active range of motion (AROM) between two different targets

We examined each method's ability to track movement in the sweep task in eight participants with chronic stroke (six males, two females). The stroke participants ranged from 41 to 80 years old, and their FMA-UE scores ranged from 13 to 20 on the days of the task. Mean sweep areas and smoothness computed with each method were similar and highly correlated ( $r$ of 0.99 and $0.91, p<0.0001$, Table 3, Figures $5 c$ and $5 f$ ). Moreover, a major goal of kinematic tracking in rehabilitation is to assess changes in motor performance over time. For the participants with repeated measurements, we also found that the changes in these measures computed between sessions were consistent and significantly correlated between the Vive and IMU approaches ( $r$ of 0.99 and $0.99, p<0.01$, Figures $5 \mathrm{~g}$ and $5 \mathrm{~h}$ ). Having sufficient sensitivity to detect changes with recovery is a key requirement for kinematic measures used in rehabilitation.

\section{Discussion}

Both IMU and HTC Vive can track wrist position in stroke survivors with relatively high accuracy and precision during multiplanar UE motion. This information can be used to derive metrics of movement quality that are clinically relevant. Compared to the expensive Vicon system, the mean errors in EPD for the IMU and Vive were both approximately $1 \mathrm{~cm}$, respectively. Mean errors in AROM were less than $2.5 \mathrm{~cm}$ for both systems. Moreover, some of the error in estimation was likely just due to variability among reaches to the same target (see Vicon data in Table 1), since these systems were not all tested in the same session. Thus, both systems may be even more accurate than these estimates. More importantly for clinical research, the IMU and Vive measured changes in AROM very accurately, differing from Vicon estimates by less than $1 \mathrm{~cm}$. Additionally, both methods estimated similar horizontal sweep areas and normalized mean endpoint speeds in stroke participants and agreed on changes in these metrics over time. Thus, both IMU and Vive offer viable options for measuring UE kinematics in clinical research settings outside the lab. In addition, we have made our code that estimates wrist position using each method publicly available (see Code Availability).

There are several potential sources for estimation error with these systems compared to optical tracking. Both the IMUs and the Vive trackers were susceptible to misalignment with the limb, as well as to changes in alignment due to soft tissue motion relative to the underlying bone. Adapting a sensor-tosegment calibration scheme to align the sensors to the bone would help minimize this source of error. However, such schemes require the subject to perform predefined static poses or movements[44,45], many of which would be difficult for moderately to severely motor-impaired stroke survivors to perform. 
This can be partially alleviated by fusing video information processed with markerless pose estimation with the IMU data[46]. For the IMU, magnetic distortion was the main source of error. Despite calibrating, the parameters were static and thus were most valid for the magnetic distortion closest to the calibration location. As the IMUs move away from the calibration location, the calibration parameters lose validity at a rate directly proportional to the degree of distortion present in the surrounding magnetic field.

We used several key methods to reduce these potential estimation error sources. These methods are described in detail in the Appendix, but the following is an introduction and general overview of these key methods. First, the kinematic models for both methods only required one of the sensor's axes to be aligned to the length of the limb. This allowed for more potential sensor attachment points around the limb's circumference and facilitated avoiding problematic soft tissue contours (e.g., adipose tissue). Additionally, the IMU-based method incorporated baseline orientation removal and passive FE motion calibration steps into its kinematic model, which helped minimize the effects of anatomic variation and sensor alignment. Furthermore, we concatenated the resting period to the beginning of the IMU recording, and we implemented an orientation solver that estimates each sensor's initial orientation. Both steps allowed for lower gain selection, which helped minimize the effects of distortion. Finally, the complementary filter algorithm itself helped reduce the impact of magnetic distortion by constraining the magnetometer to only influence heading (i.e. yaw) $[24,28]$.

Although our results show that both the IMU and Vive track wrist position with comparable accuracy, each method has important advantages and disadvantages to consider. Regarding setup and usability, the IMU's magnetometer requires calibration for accurate tracking, especially if the sensors are used in different environments. In addition, the IMU-based method requires a resting period and flexion-extension movements, which combined require approximately a minute to perform, prior to starting a task. These steps are not necessary when using the Vive. However, the Vive lighthouses require careful placement to avoid occlusion of the trackers. This may not a problem for arm motion tracking in stroke survivors since the required tracking area is small, as it took us approximately a minute to properly place the lighthouses in the laboratory, but may be a consideration depending on the task and environment (e.g., may take a bit longer to set up lighthouses in a hospital room). Also, the Vive trackers are bulkier and slightly heavier than the IMUs, which may make them less suitable in stroke survivors with severe arm weakness or in tighter spaces. Both the IMU and Vive may be suitable for hospital rooms, but the Vive may be better if the environment is less controllable in terms of metal objects. Finally, the IMU used in this study can record electromyography data synchronously, which could be advantageous for some studies.

In addition to the extensive setup and calibration described in the Introduction, we found that small, occasionally trivial disturbances in the setup, such as slamming a door or accidently bumping a reflective marker, adversely affected Vicon estimations and necessitated recalibration. These disadvantages, as well as the difficulty of transporting the hardware itself, makes routine use of the Vicon in different locations challenging. Compared to the Vicon and other optical tracking systems, relatively minimal alignment and calibration steps are required, especially for the Vive, and the calibration motions were achievable by stroke survivors. This made motion tracking less burdensome on the patient and more 
convenient for the researcher or clinician. Furthermore, both systems are compact, lightweight, and relatively easy to set up. This permits UE motion tracking for patients outside of the laboratory environment, such as in an inpatient setting early after stroke. Finally, our IMUs and Vive are far less expensive than traditional optical tracking systems, or commercial IMU systems with software dedicated to motion tracking (e.g. Xsens), making them more accessible to researchers and clinicians, especially in underdeveloped countries.

There has been a recent call for more routine use of kinematic measures in stroke trials, as well as for more evidence of the reliability and accuracy of kinematic measurement systems[11]. However, this remains a challenge because of the paucity of validated methods that can practically measure kinematics at bedside. Our paper attempts to address this gap in the literature. Moreover, in addition to making our Vive-based method's code open-source, this is the first study to investigate using the Vive as a method of estimating 3D wrist position in stroke survivors to our knowledge. Several studies have used VR systems as the intervention in conjunction with measuring kinematics in stroke[13-16], but not as the method of measuring kinematics. Similar to ours, one of these studies measured kinematics by creating a kinematic model using IMUs[14], but they did not evaluate the accuracy of their method and used Madgwick's gradient descent algorithm[32] to compute IMU orientation. This algorithm, despite several merits, has several disadvantages that make it more susceptible to measurement distortion and is the predecessor to Madgwick's improved algorithm[28], which we used in our study. Another study utilized a kinematic model created by IMUs to assess movement kinematics in healthy and stroke participants performing the ARAT, but they did not validate the accuracy of their sensor fusion algorithm and kinematic model using a gold standard[47]. A third study utilized IMUs to study kinematic synergies of UE movement in healthy participants, but these sensors were from a commercial system with software dedicated to motion tracking [48]. In contrast, we evaluated the accuracy of our IMU-based method against a gold standard Vicon. Moreover, we have made our code for the IMU-based method open-source, which can be easily adapted to work with any generic 9-axis IMU.

An emerging method for markerless vision-based pose estimation is the use of deep neural networks to estimate pose from 2D images[22,23,49,50], which could be promising for clinical environments. One study utilized this method in a hospital setting, but it only measured 2D pose and required manually labeled training data in each patient to account for environmental variations and to ensure proper tracking of a broad range of postures[23]. Further, only one study tracked 3D pose using a stereoscopic camera system in a laboratory environment, but accuracy was not rigorously evaluated[22]. These studies did not directly evaluate accuracy in terms of Cartesian space (e.g., in cm), making the accuracy results less clinically relevant. Finally, these techniques are also subject to potential occlusion, depending on the type of motion made. Thus, it remains to be seen how markerless motion tracking will compare to attachable external sensors in terms of performance, as well as cost.

This study has several limitations. First, despite carefully designing and executing the reaching task, the Vive was compared to the Vicon asynchronously due to hardware incompatibilities, precluding a rigorous comparison of accuracy when performing tasks at different speed. Also, these methods were only tested 
in a laboratory environment. Moreover, it is worth highlighting that there is additional kinematic information estimated that we did not analyze in this study, including elbow angle and position. Future work will include evaluating these methods in a hospital environment, incorporating a sensor-to-segment calibration scheme compatible with motor-impaired stroke survivors, and studying more clinically relevant kinematic metrics using these methods.

Kinematic outcome metrics are an important, emerging technique in stroke rehabilitation, which may provide more sensitive and specific outcome measures than commonly used clinical scores. Importantly, these methods can remove confounds from compensatory movements that may distort functional scores. Although not the end goal of this study, we showed two examples of functionally relevant kinematic metrics that can be derived from the methods, the sweep area and smoothness. These methods can be extended to estimate numerous other kinematic metrics relevant to stroke survivors[8], for example, wrist linear and elbow angular velocities, accelerations, and jerks. It remains to be seen what types of kinematic metrics will be most useful for identifying improvement after stroke rehabilitation.

\section{Conclusion}

Measuring kinematics is an emerging technique to measure outcomes from stroke rehabilitation and is achievable by estimating pose. Traditional marker-based optical tracking systems are considered the gold-standard for pose estimation. However, they require extensive setup and calibration, making them suboptimal to use in motor-impaired stroke patients, especially in clinical environments. In this study, we presented and demonstrated the tracking accuracy and sensitivity of two portable methods utilizing wearable sensors for pose estimation. We also demonstrated these methods' ability to estimate pose in chronic stroke survivors with relative ease. This work provides two validated, open-source methods that can be used to estimate kinematics practically and accurately in motor-impaired stroke survivors in clinical environments.

\section{Abbreviations}

ARAT: Action Research Arm Test; FMA-UE: Fugl-Meyer Assessment of Upper Extremity; IMU: Inertial measurement unit; VR: Virtual reality; UE: Upper extremity; FE: Flexion-extension; PS: Pronation-supination; EPD: Endpoint distance; AROM: Active range of motion; EPS: Endpoint speed; $\triangle A R O M$ : Changes in active range of motion

\section{Declarations}

\section{Code Availability}

The code used to estimate wrist position with respect to the shoulder using the IMUs and Vive is available at https://github.com/JeffNie96/IMU-Vive-Kinematics. Please contact J.Z.N. via email with any inquiries.

\section{Ethics approval and consent to participate}


All participants were admitted to the study following informed consent or assent as approved by the Northwestern University Institutional Review Board.

\section{Consent for publication}

All participants were informed about the study and signed a consent form or assented.

\section{Availability of data and materials}

The code used to estimate wrist position using the two methods are available at https://github.com/JeffNie96/IMU-Vive-Kinematics. The datasets used and/or analyzed during the current study are available from the corresponding author on reasonable request.

\section{Competing interests}

The authors declare that they have no competing interests

\section{Funding}

This paper was funded by the National Institute of Neurological Disorders and Stroke of the National Institutes of Health under Award Numbers [R01NS099210] and [R01NS112942].

\section{Authors' contributions}

J.Z.N. designed the study, wrote most of the code, collected and analyzed the data, and wrote the majority manuscript. J.W.N. helped design the study, wrote some of the code, collected the data, and contributed to the manuscript. N.H. extracted Vive estimations from Unity, collected data, and contributed to the manuscript. R.J.C. collected data and contributed to the manuscript. M.W.S. helped design the study and contributed to the manuscript. M.W.S. is the Principal Investigator of this study.

\section{Acknowledgements}

The authors would like to thank Vivek Paul and Prasanth Ravi Prakash for their assistance with data collection for this study.

\section{Authors' information}

${ }^{1}$ Southern Illinois University School of Medicine, Springfield, IL 62794, United States of America

${ }^{2}$ Department of Neurology, Northwestern University, Chicago, IL 60611, United States of America

${ }^{3}$ University of Illinois at Chicago College of Medicine, Chicago, IL 60612, United States of America

${ }^{4}$ Shirley Ryan AbilityLab, Chicago, IL 60611, United States of America 
${ }^{5}$ Department of Physical Medicine and Rehabilitation, Northwestern University, Chicago, IL 60611, United States of America

${ }^{6}$ Department of Physiology, Northwestern University, Chicago, IL 60611, United States of America

${ }^{7}$ Department of Biomedical Engineering, Northwestern University, Chicago, IL 6061, United States of America

\section{References}

1. Virani SS, Alonso A, Benjamin EJ, Bittencourt MS, Callaway CW, Carson AP, et al. Heart disease and stroke statistics-2020 update: A report from the American Heart Association. Circulation. 2020;141:e139-596.

2. Hatem SM, Saussez G, della Faille M, Prist V, Zhang X, Dispa D, et al. Rehabilitation of Motor Function after Stroke: A Multiple Systematic Review Focused on Techniques to Stimulate Upper Extremity Recovery. Front Hum Neurosci. 2016;10.

3. Salter K, Campbell N, Richardson M, Mehta S, Jutai J, Zettler L, et al. Outcome Measures in Stroke Rehabilitation. Evidence-Based Rev Stroke Rehabil. 2013.

4. Fugl-Meyer AR, Jääskö L, Leyman I, Olsson S, Steglind S. The post-stroke hemiplegic patient. 1. a method for evaluation of physical performance. Scand J Rehabil Med. 1975;7:13-31.

5. Lyle RC. A performance test for assessment of upper limb function in physical rehabilitation treatment and research. Int. J. Rehabil. Res. 1981. p. 483-92.

6. Van Dokkum L, Hauret I, Mottet D, Froger J, Métrot J, Laffont I. The contribution of kinematics in the assessment of upper limb motor recovery early after stroke. Neurorehabil Neural Repair. 2014;28:412.

7. Krakauer JW. Motor learning: Its relevance to stroke recovery and neurorehabilitation. Curr Opin Neurol. 2006;19:84-90.

8. Schwarz A, Kanzler CM, Lambercy O, Luft AR, Veerbeek JM. Systematic Review on Kinematic Assessments of Upper Limb Movements After Stroke. Stroke. 2019;50:718-27.

9. Thrane G, Alt Murphy M, Sunnerhagen KS. Recovery of kinematic arm function in well-performing people with subacute stroke: A longitudinal cohort study. J Neuroeng Rehabil. Journal of NeuroEngineering and Rehabilitation; 2018;15:1-10.

10. Murphy MA, Willén $C$, Sunnerhagen KS. Kinematic variables quantifying upper-extremity performance after stroke during reaching and drinking from a glass. Neurorehabil Neural Repair. 2011;25:71-80.

11. Kwakkel G, van Wegen EEH, Burridge JH, Winstein CJ, van Dokkum LEH, Alt Murphy M, et al. Standardized Measurement of Quality of Upper Limb Movement After Stroke: Consensus-Based Core Recommendations From the Second Stroke Recovery and Rehabilitation Roundtable. Neurorehabil Neural Repair. 2019;33:951-8. 
12. Mugler EM, Tomic G, Singh A, Hameed S, Lindberg EW, Gaide J, et al. Myoelectric Computer Interface Training for Reducing Co-Activation and Enhancing Arm Movement in Chronic Stroke Survivors: A Randomized Trial. Neurorehabil Neural Repair. 2019;33:284-95.

13. Hussain N, Sunnerhagen KS, Alt Murphy M. End-point kinematics using virtual reality explaining upper limb impairment and activity capacity in stroke. J Neuroeng Rehabil. Journal of NeuroEngineering and Rehabilitation; 2019;16:1-9.

14. Wittmann F, Held JP, Lambercy O, Starkey ML, Curt A, Höver R, et al. Self-directed arm therapy at home after stroke with a sensor-based virtual reality training system. J Neuroeng Rehabil. Journal of NeuroEngineering and Rehabilitation; 2016;13:1-10.

15. Subramanian SK, Lourenço CB, Chilingaryan G, Sveistrup H, Levin MF. Arm motor recovery using a virtual reality intervention in chronic stroke: Randomized control trial. Neurorehabil Neural Repair. 2013;27:13-23.

16. Liebermann DG, Berman S, Weiss PL, Levin MF. Kinematics of reaching movements in a 2-D virtual environment in adults with and without stroke. IEEE Trans Neural Syst Rehabil Eng. 2012;20:778-87.

17. Zhou $\mathrm{H}, \mathrm{Hu} \mathrm{H}$. Human motion tracking for rehabilitation-A survey. Biomed Signal Process Control. 2008;3:1-18.

18. Carse B, Meadows B, Bowers R, Rowe P. Affordable clinical gait analysis: An assessment of the marker tracking accuracy of a new low-cost optical 3D motion analysis system. Physiotherapy. The Chartered Society of Physiotherapy; 2013;99:347-51.

19. Windolf M, Götzen N, Morlock M. Systematic accuracy and precision analysis of video motion capturing systems-exemplified on the Vicon-460 system. J Biomech. 2008;41:2776-80.

20. Upper Limb Model Product Guide. Vicon Motion Systems Limited; 2007.

21. Vicon Nexus Product Guide. Vicon Motion Systems Limited; 2015.

22. Arac A, Zhao P, Dobkin BH, Carmichael ST, Golshani P. Deepbehavior: A Deep Learning Toolbox for Automated Analysis of Animal and Human Behavior Imaging Data. Front Syst Neurosci. 2019;13.

23. Chen K, Gabriel P, Alasfour A, Gong C, Doyle WK, Devinsky O, et al. Patient-specific pose estimation in clinical environments. IEEE J TransI Eng Heal Med. IEEE; 2018;6.

24. Cotton RJ, Rogers J. Wearable Monitoring of Joint Angle and Muscle Activity. Proc 16th IEEE Int Conf Rehabil Robot. Toronto, Canada; 2019. p. 258-63.

25. Sabatini AM. Estimating Three-Dimensional Orientation of Human Body Parts by Inertial/Magnetic Sensing. Sensors. 2011;11:1489-525.

26. Roetenberg D, Luinge H, Slycke P. Xsens MVN: Full 6DOF Human Motion Tracking Using Miniature Inertial Sensors. Enschede; 2013.

27. Bertomeu-Motos A, Lledó LD, Díez JA, Catalan JM, Ezquerro S, Badesa FJ, et al. Estimation of Human Arm Joints Using Two Wireless Sensors in Robotic Rehabilitation Tasks. Sensors. 2015;15:3057183. 
28. Fan B, Li Q, Liu T. How Magnetic Disturbance Influences the Attitude and Heading in Magnetic and Inertial Sensor-Based Orientation Estimation. Sensors. 2018;18.

29. Valenti RG, Dryanovski I, Xiao J. Keeping a Good Attitude: A Quaternion-Based Orientation Filter for IMUs and MARGs. Sensors. 2015;15:19302-30.

30. Wu J, Zhou Z, Chen J, Fourati H, Li R. Fast Complementary Filter for Attitude Estimation Using LowCost MARG Sensors. IEEE Sens J. IEEE; 2016;16:6997-7007.

31. Seel T, Schauer T, Raisch J. Joint Axis and Position Estimation from Inertial Measurement Data by Exploiting Kinematic Constraints. Proc from 2012 IEEE Int Conf Control Appl. Dubrovnik, Croatia; 2012. p. 45-9.

32. Madgwick SOH, Harrison AJL, Vaidyanathan R. Estimation of IMU and MARG orientation using a gradient descent algorithm. Proc from 2011 IEEE Int Conf Rehabil Robot. IEEE; 2011;1-7.

33. de Vries WHK, Veeger HEJ, Baten CTM, van der Helm FCT. Magnetic distortion in motion labs, implications for validating inertial magnetic sensors. Gait Posture. 2009;29:535-41.

34. van der Veen SM, Bordeleau M, Pidcoe PE, France CR, Thomas JS. Agreement analysis between vive and vicon systems to monitor lumbar postural changes. Sensors. 2019;19.

35. Li Q, Griffiths JG. Least Squares Ellipsoid Specific Fitting. Geom Model Process 2004, Proc. Beijing, China; 2004. p. 335-40.

36. Afzal MH. Complete Triaxis Magnetometer Calibration in the Magnetic Domain. J Sensors. 2010;2010.

37. Tomczynski J, Mankowsi T, Kaczmarek P. Cross-Sensor Calibration Procedure for Magnetometer and Inertial Units. In: Szewczyk R, Zieliński C, Kaliczyńska M, editors. Adv Intell Syst Comput. Springer, Cham; 2017. p. 450-9.

38. Borges M, Symington A, Coltin B, Smith T, Ventura R. HTC Vive: Analysis and Accuracy Improvement. 2018 IEEE/RSJ Int Conf Intell Robot Syst. Madrid, Spain; 2018. p. 2610-5.

39. Hung N-T, Paul V, Kovach T, Slutzky MW. Wearable Myoelectric Interface Training for Improving Arm Movement in Chronic Stroke. Proc Int Stroke Conf. 2021.

40. McPherson JG, Chen A, Ellis MD, Yao J, Heckman CJ, Dewald JPA. Progressive recruitment of contralesional cortico-reticulospinal pathways drives motor impairment post stroke. J Physiol. 2018;596:1211-25.

41. Rohrer B, Fasoli S, Krebs HI, Hughes R, Volpe B, Frontera WR, et al. Movement smoothness changes during stroke recovery. J Neurosci. 2002;22:8297-304.

42. Krebs HI, Hogan N, Aisen ML, Volpe BT. Robot-Aided Neurorehabilitation. IEEE Trans Rehabil Eng. 1998;6:75-87.

43. Holoborodko P. Smooth Noise-Robust Differentiators [Internet]. 2008 [cited 2020 Dec 23]. Available from: http://www.holoborodko.com/pavel/numerical-methods/numerical-derivative/smooth-lownoise-differentiators/ 
44. Choe N, Zhao H, Qiu S, So Y. A sensor-to-segment calibration method for motion capture system based on low cost MIMU. Measurement. 2019;131:490-500.

45. Zabat M, Ababou A, Ababou N, Dumas R. IMU-based sensor-to-segment multiple calibration for upper limb joint angle measurement-a proof of concept. Med Biol Eng Comput. Medical \& Biological Engineering \& Computing; 2019;57:2449-60.

46. Cotton RJ. Kinematic Tracking of Rehabilitation Patients With Markerless Pose Estimation Fused with Wearable Inertial Sensors. Proc 15th IEEE Int Conf Autom Face Gesture Recognit. 2020. p. 50814.

47. Repnik E, Puh U, Goljar N, Munih M, Mihelj M. Using inertial measurement units and electromyography to quantify movement during action research arm test execution. Sensors. 2018;18:1-23.

48. Tang S, Chen L, Barsotti M, Hu L, Li Y, Wu X, et al. Kinematic synergy of multi-DOF movement in upper limb and its application for rehabilitation exoskeleton motion planning. Front Neurorobot. 2019;13.

49. Mathis A, Mamidanna P, Cury KM, Abe T, Murthy VN, Mathis MW, et al. DeepLabCut: markerless pose estimation of user-defined body parts with deep learning. Nat Neurosci. Springer US; 2018;21:12819.

50. Toshev A, Szegedy C. DeepPose: Human Pose Estimation via Deep Neural Networks. 2014 IEEE Conf Comput Vis Pattern Recognit. 2014. p. 1653-60.

51. Diebel J. Representing Attitude: Euler Angles, Unit Quaternions, and Rotation Vectors. Stanford; 2006.

52. Wahba G. Problem 65-1: A Least Squares Estimate of Satellite Attitude. SIAM Rev. 2007;7:409.

53. Shuster MD, Oh SD. Three-Axis Attitude Determination from Vector Observations. J Guid Control Dyn. 1981;4:70-7.

54. Markley FL. Fast Quaternion Attitude Estimation from Two Vector Measurements. J Guid Control Dyn. 2002;25:411-4.

55. National Oceanic and Atmospheric Administration. World Magnetic Model [Internet]. Available from: https://www.ngdc.noaa.gov/geomag/calculators/magcalc.shtml\#igrfwmm

56. Waldron K, Schmiedeler J. Kinematics. In: Siciliano B, Khatib O, editors. Springer Handb Robot. 2nd ed. Springer, Berlin, Heidelberg; 2016. p. 9-33.

\section{Appendix}

The Appendix is available in the Supplemental Files section

\section{Figures}


a

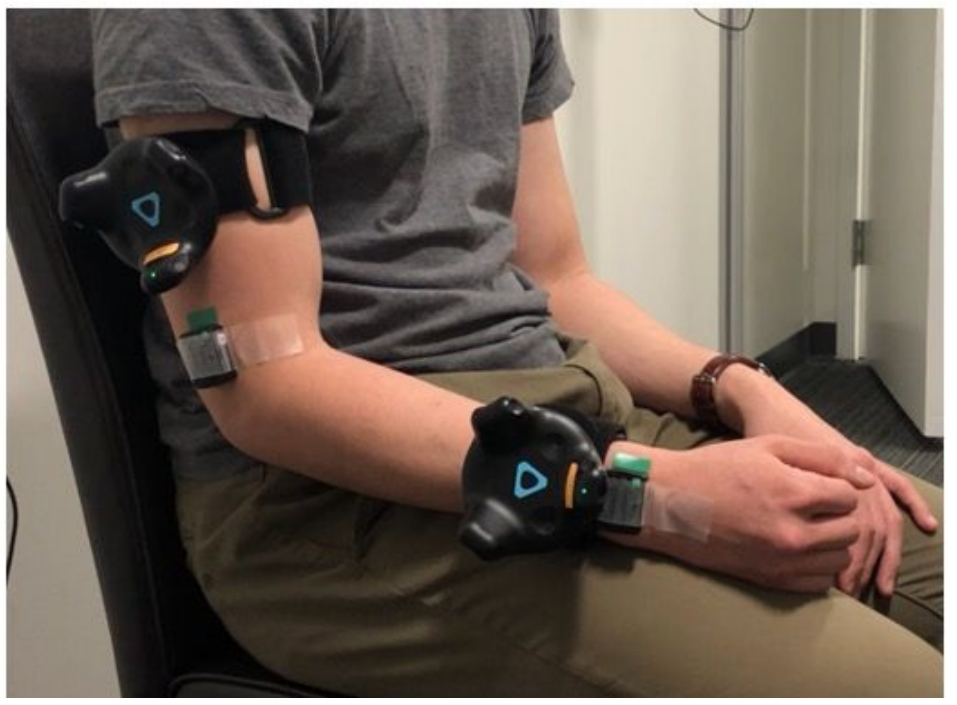

b

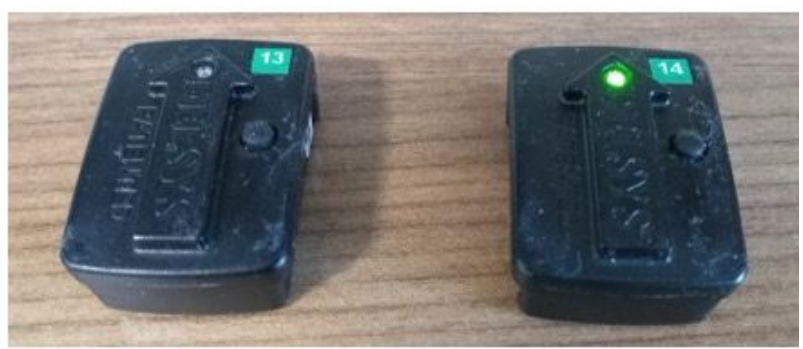

C

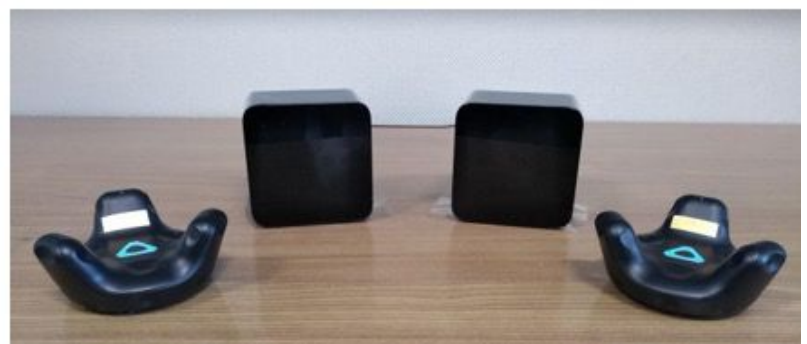

\section{Figure 1}

Sensors used to estimate elbow angle and wrist position. a. The 9-axis Trigno IMUs, which also record electromyography data. b. The Vive trackers (outside) and lighthouses (middle). c. An example IMU and Vive setup on a human participant.

a

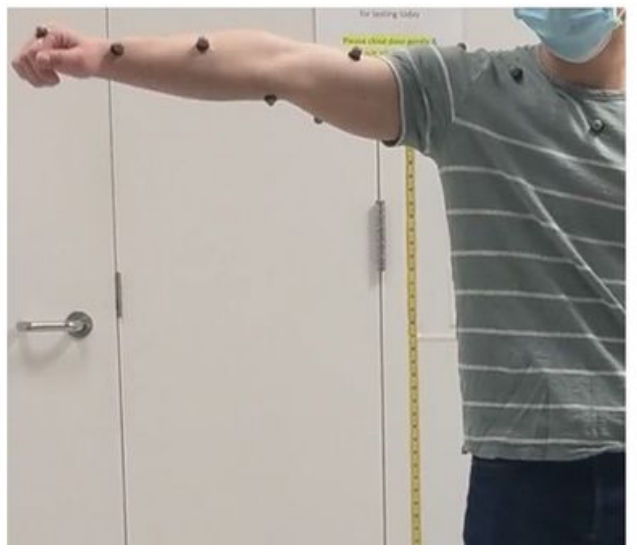

b

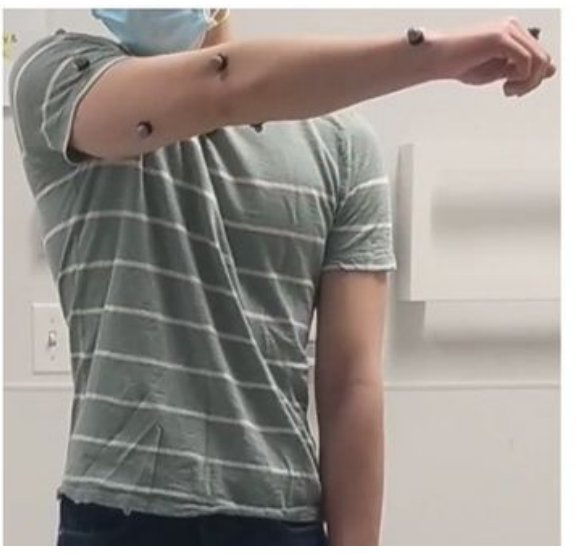

C

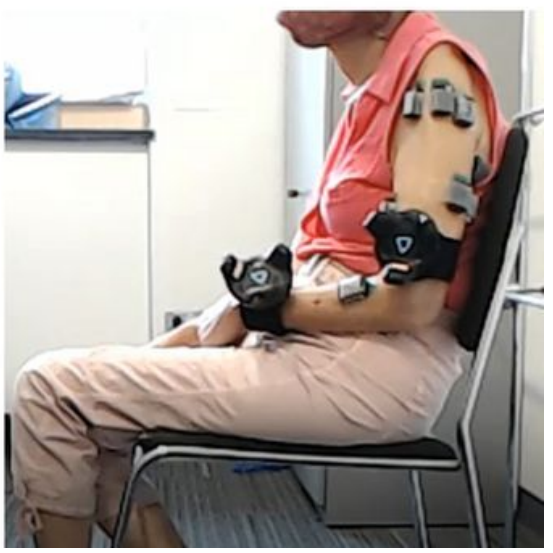

\section{Figure 2}

Vicon upper extremity (UE) marker setup and an example sensor setup on a stroke patient. a. Vicon marker placements on the anterior arm, anterior forearm, and radial wrist. b. Vicon marker placement on the posterior arm and ulnar wrist. c. The IMU and Vive setup on a stroke participant. The additional sensors on the UE are electromyography sensors. 

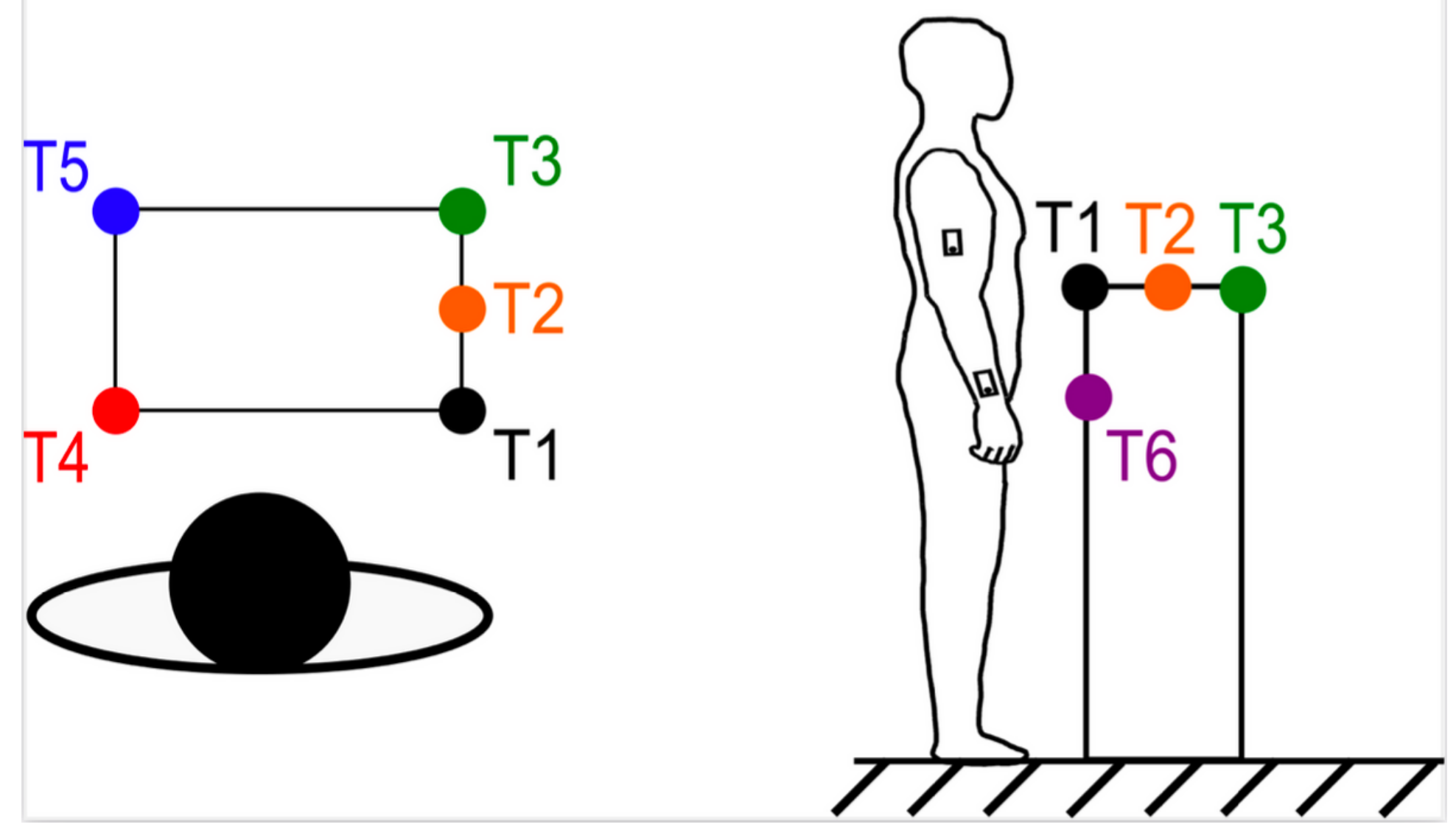

Figure 3

Schematic of the first task. Starting with the arm at the side, a healthy participant reached out towards target one (T1), held the reach for several seconds, then returned the arm to the side. The participant repeated this movement sequence for the remaining targets (T2 to T6) in sequential order. 


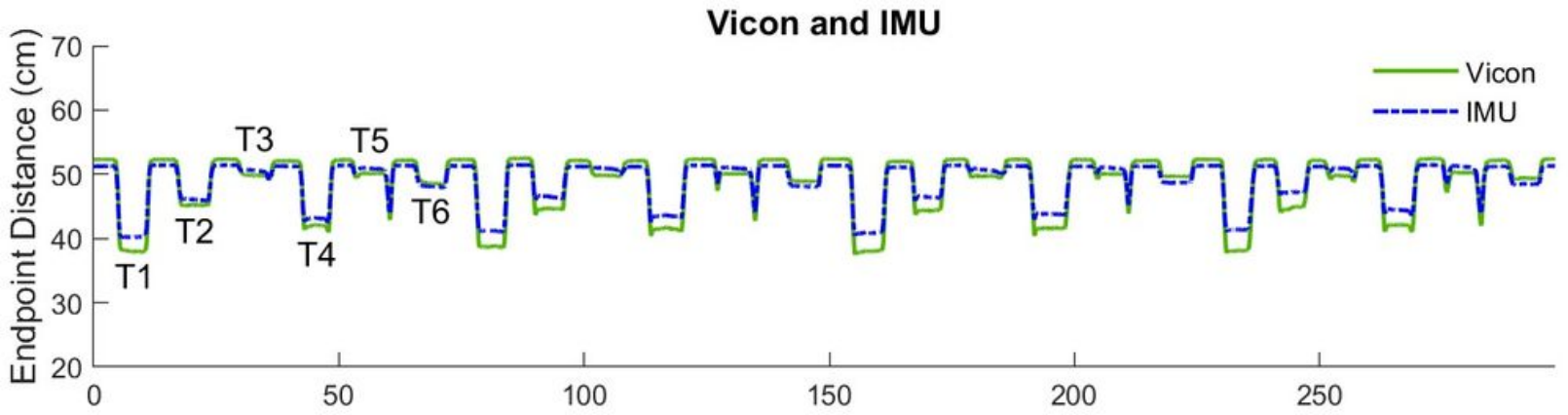

b

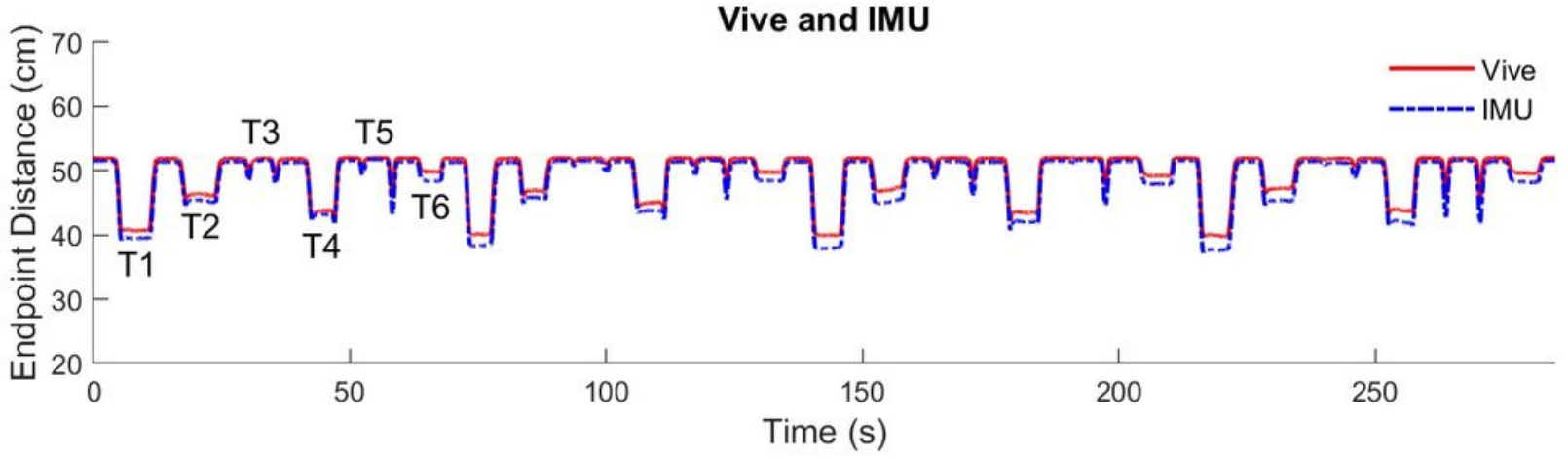

Figure 4

Example of end-point distance (EPD) tracking during the first task from the Vicon, IMU, and Vive. T1 to T6 refer to targets 1 to 6 , respectively. a, EPD estimations from four reaches to each target (24 reaches total) while recording from the Vicon and IMUs synchronously. b, EPD estimations from four reaches to each target while recording from the Vive and IMUs synchronously. 

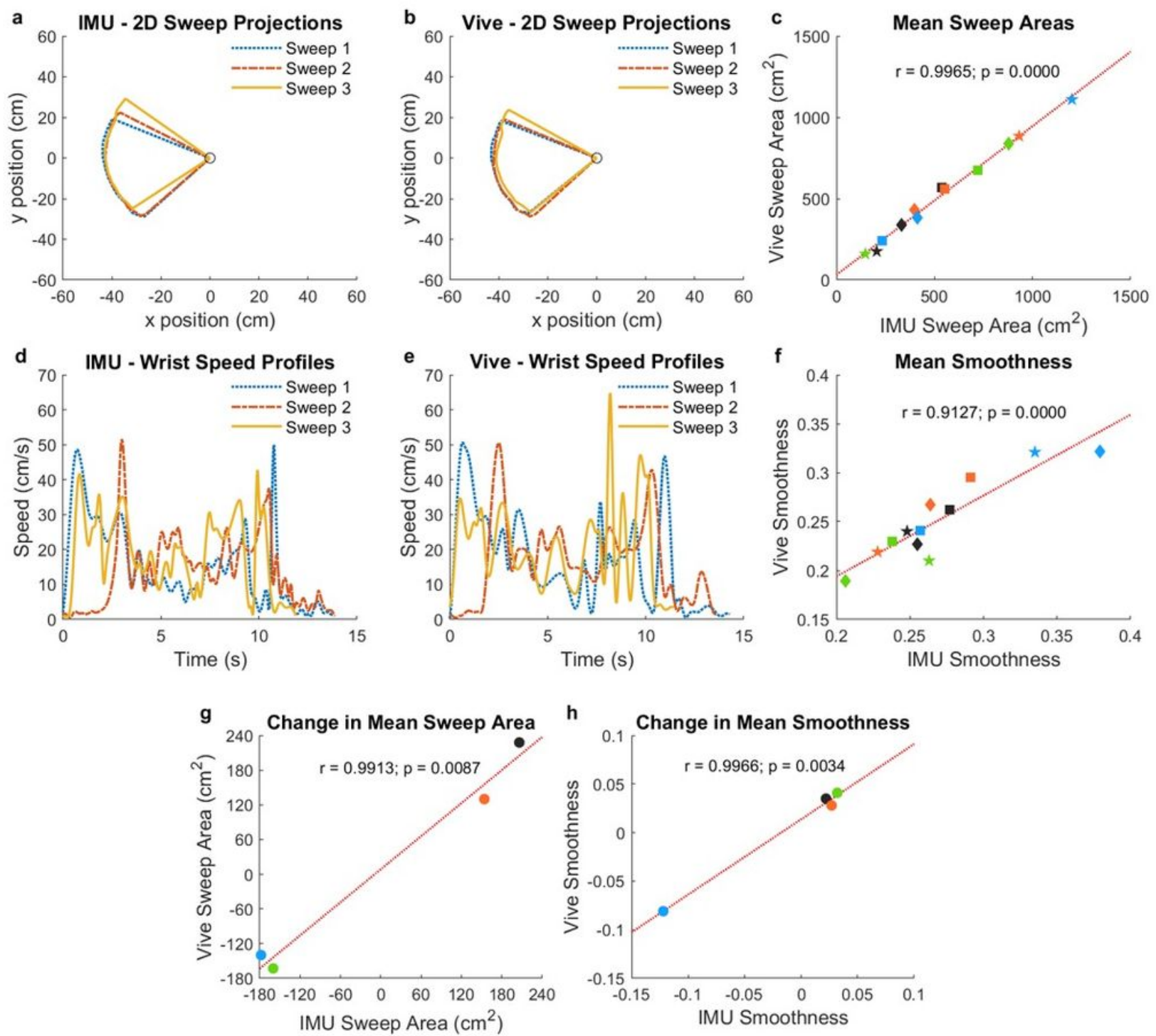

\section{Figure 5}

Sweep task kinematic metrics. a. Example traces of the sweep areas estimated by the IMUs in participant 8 (P8). The three sweeps (blue, red, yellow) were projected onto the horizontal plane. b. Example traces of the sweep areas estimated by the Vive. c. Estimated mean sweep areas for all sweep tasks performed by all participants. Each marker is one mean sweep area. Black - P1 (diamond - baseline week, square later week) and P5 (star); orange - P2 (diamond, square) and P6 (star); green - P3 (diamond, square) and P7 (star); blue - P4 (diamond, square) and P8 (star). d. Example traces of wrist speed profiles estimated by the IMUs. e. Example traces of the wrist speed estimated by the Vive. f. Estimated smoothness for all sweep tasks. g. Change in mean sweep area between the baseline and later weeks for P1, P2, P3, and P4. Black - P1; orange - P2; green - P3, blue - P4. h. Change in mean smoothness between the baseline and later weeks. 


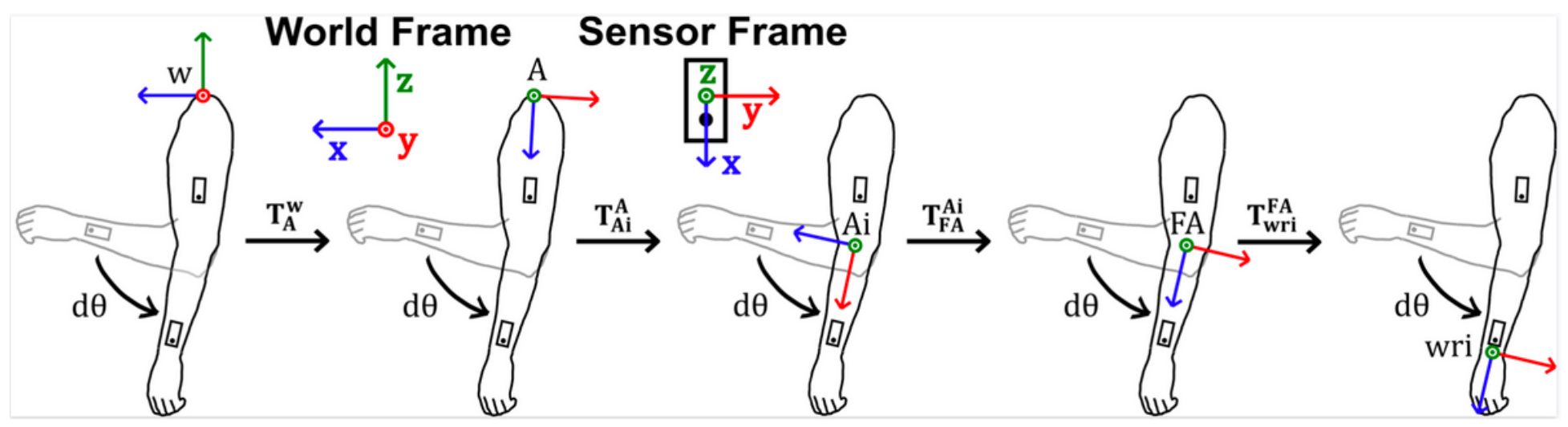

Figure 6

Sequence of four transformations for the IMUs that transforms a world frame (w, left) with origin at the shoulder to a frame aligned with the forearm with origin at the wrist (wri, right). The gray limbs represent the limb orientations when the baseline orientation difference was removed (i.e. initial orientation), and the darker limbs represent an extension of the elbow by $\mathrm{d} \theta$ from the initial state. A, true arm frame (aligned with the arm sensor); Ai, intermediate arm frame (aligned with the forearm sensor initially); FA, true forearm frame (aligned with the forearm sensor); $T$, transformation matrices.

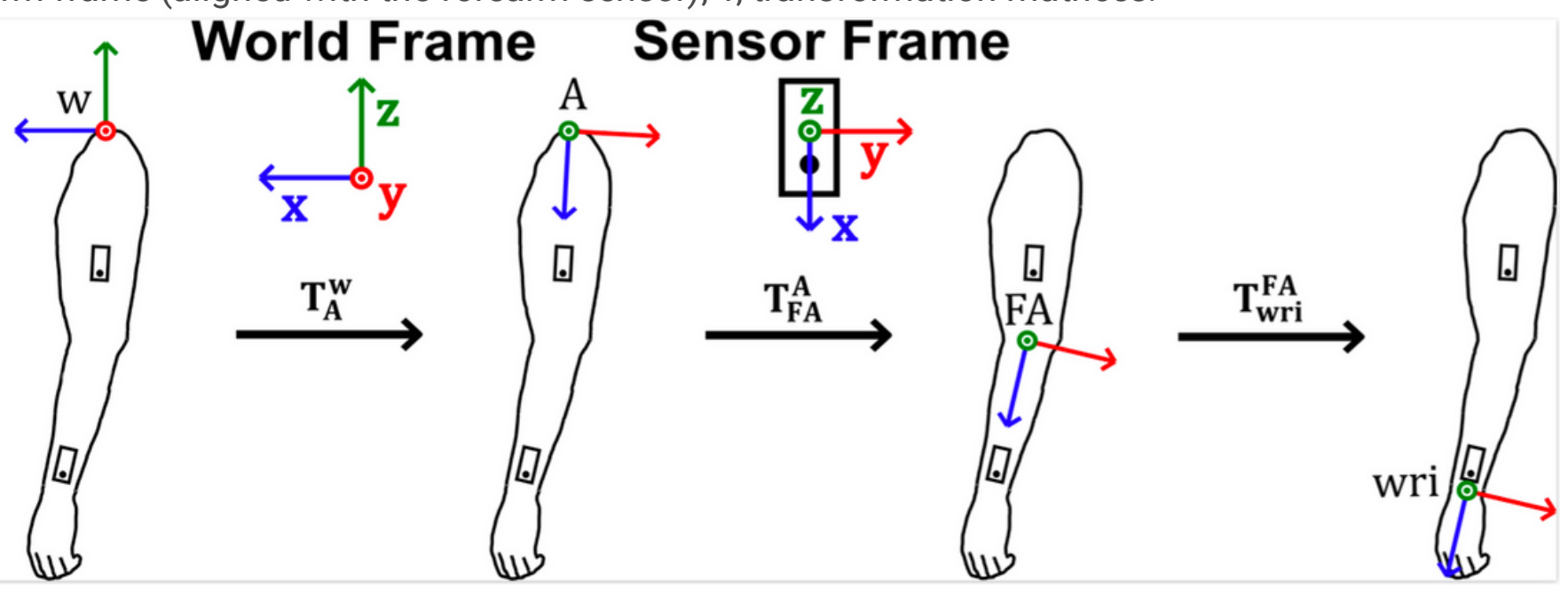

\section{Figure 7}

Sequence of three transformations for the Vive that transforms a world frame ( $w$, left) with origin at the shoulder to a frame aligned with the forearm with origin at the wrist (wri, right). A, true arm frame (aligned with the arm sensor); FA, true forearm frame (aligned with the forearm sensor); $T$, transformation matrices.

\section{Supplementary Files}

This is a list of supplementary files associated with this preprint. Click to download.

- Appendix.docx 